\title{
Early use of donepezil against psychosis and cognitive decline in Parkinson's disease: a randomised controlled trial for 2 years
}

\author{
Hideyuki Sawada, ${ }^{1}$ Tomoko Oeda, ${ }^{1}$ Masayuki Kohsaka, ${ }^{1}$ Atsushi Umemura, ${ }^{1}$ \\ Satoshi Tomita, ${ }^{1}$ Kwiyoung Park, ${ }^{1}$ Kouichi Mizoguchi, ${ }^{2}$ Hidenori Matsuo, ${ }^{3}$ \\ Kazuko Hasegawa, ${ }^{4}$ Harutoshi Fujimura, ${ }^{5}$ Hiroshi Sugiyama, ${ }^{1,6}$ Michikazu Nakamura, ${ }^{7}$ \\ Seishi Kikuchi, ${ }^{8}$ Kenji Yamamoto, ${ }^{1}$ Toshiaki Fukuda, ${ }^{1}$ Suminobu Ito, ${ }^{9}$ Masashi Goto, ${ }^{10}$ \\ Kosuke Kiyohara, ${ }^{11}$ Takashi Kawamura ${ }^{12}$
}

\begin{abstract}
- Additional material is published online only. To view please visit the journal online (http://dx.doi.org/10.1136/ jnnp-2018-318107)

For numbered affiliations see end of article.
\end{abstract}

\section{Correspondence to} Dr Hideyuki Sawada, Clinical Research Center and Department of Neurology, Utano National Hospital, Kyoto 6168255, Japan; sawada. unh@gmail.com

Received 28 January 2018 Revised 27 April 2018 Accepted 10 July 2018 Published Online First 3 August 2018

\section{Check for updates}

(c) Author(s) (or their employer(s)) 2018. Re-use permitted under CC BY-NC. No commercial re-use. See rights and permissions. Published by BMJ.

To cite: Sawada H, Oeda T, Kohsaka M, et al. J Neurol Neurosurg Psychiatry 2018:89:1332-1340.

\section{ABSTRACT}

Objectives Brain acetylcholine is decreased even in patients with cognitively preserved Parkinson's disease (PD). We investigated whether early and long-term use of donepezil prevents psychosis in non-demented PD patients.

Methods A double-blinded, placebo-controlled trial was conducted. A total of 145 non-demented PD patients were randomly assigned to receive $5 \mathrm{mg} /$ day donepezil $(n=72)$ or placebo $(n=73)$ for 96 weeks. Medications for PD were not restricted, but antipsychotic drugs were not permitted throughout the study.

The primary outcome measure was survival time to psychosis that was predefined by Parkinson's Psychosis Questionnaire (PPQ) B score $\geq 2$ or C score $\geq 2$. Secondary outcome measures included psychosis developing within 48 weeks, total PPQ score, Mini-Mental State Examination (MMSE), Wechsler Memory Scale (WMS) and subgroup analysis by apolipoprotein $\varepsilon 4$ genotyping. Results Kaplan-Meier curves for psychosis development were very similar between the two groups, and the Cox proportional hazard model revealed an adjusted HR of $0.87(95 \% \mathrm{Cl} 0.48$ to 1.60$)$. The changes in MMSE and WMS-1 (auditory memory) were significantly better with donepezil than in placebo. In the subgroup analysis, donepezil provided an HR of $0.31(0.11-0.86)$ against psychosis in 48 weeks for apolipoprotein $\varepsilon 4$ non-carriers. Conclusions Although donepezil provided beneficial effects on PPQ, MMSE and auditory WMS score changes in 2 years, it had no prophylactic effect on development of psychosis in PD. Apolipoprotein $\varepsilon 4$ may suppress the antipsychotic effect of donepezil.

Trial registration number UMIN000005403.

\section{INTRODUCTION}

Prophylaxis against psychosis and cognitive decline is an unmet need in Parkinson's disease (PD). During long-term medical treatment of motor symptoms and signs, patients with PD often develop psychosis, with a reported prevalence of about $10 \%-40 \%,{ }^{1-3}$ and psychosis is one of the most common non-motor symptoms. ${ }^{4}$ While motor symptoms and signs develop gradually and usually progress steadily, psychosis often occurs unexpectedly, even in early stages of the disease or in non-demented patients. ${ }^{6}$
Cognitive decline is also an important issue in longterm treatment, and most patients suffer from it in advanced stages.

Antagonists to dopaminergic receptors are efficacious against current psychosis in PD, but they are known to worsen motor symptoms and signs. ${ }^{7}$ Clozapine, a dopamine antagonist with a partial antagonistic activity to the serotonin 5HT-2A receptor, improves psychosis without worsening extrapyramidal signs, but its clinical use is restricted owing to potential agranulocytosis. ${ }^{8} 9$ In placebo-controlled randomised trials, other antidopaminergic drugs such as quetiapine and olanzapine, which are structurally similar to clozapine, have not proved efficacious against psychosis. Olanzapine improves psychosis, but its effectiveness is not superior to placebo and, in addition, it significantly worsens PD motor symptoms. ${ }^{10}$ Although quetiapine has been reported to be efficacious against PD psychosis in some case reports, randomised controlled trials show that it provides no significant benefits compared with placebo. ${ }^{11-13}$ Another drug, risperidone, improves psychosis, but it worsens motor symptoms. ${ }^{14}$ In addition to dopamine, serotonin is also associated with psychosis in PD. A serotonin 5HT-3 receptor antagonist, ondansetron, can improve psychotic symptoms, ${ }^{15}$ and pimavanserin, a 5HT-2 inverse agonist, improves psychosis ${ }^{16}{ }^{17}$; however, it is uncertain whether these drugs provide prophylaxis against PD psychosis.

Previous studies demonstrated degeneration of cholinergic neurons in PD brains, in addition to degeneration of dopaminergic neurons, ${ }^{18} 19$ and cholinergic neuronal degeneration is associated with the development of psychosis and cognitive disturbance. ${ }^{20} 21$ In addition, the use of central anticholinergic drugs is strongly associated with the development of psychosis and cognitive disturbance. ${ }^{22} 23$ The main hypothesis of the present study was that pharmacological reinforcement of cholinergic neuronal transmission would provide prophylaxis against psychosis, and in addition, beneficial effects on cognitive function in non-demented PD patients.

Donepezil is reported to ameliorate psychotic symptoms in dementia with Lewy bodies (DLB), ${ }^{24}$ but it is unclear whether donepezil would provide 
beneficial effects in non-demented PD patients. PD and DLB have commonality in their pathological changes and cholinergic neuronal destruction, and the activity of brain acetylcholinesterase is relatively preserved in non-demented PD patients compared with patients with PD dementia or DLB. ${ }^{25}$ In this context, larger pharmacological responses to cholinesterase inhibitors were expected in patients without dementia than those with dementia, and it was thought that early and long-term use may provide beneficial effects against psychosis development and cognitive decline in PD.

\section{METHODS}

\section{Study design}

The efficacy of donepezil against psychosis in PD Efficacy of Donepezil Against Psychosis (EDAP) study was a doubleblinded, placebo-controlled, parallel-armed, randomised trial that was performed as a physician-initiated study for approval based on the Pharmaceutical Affairs Law, between May 2011 and November 2013, at eight hospitals of the National Hospital Organization, Japan. The core of the study protocol was published previously. ${ }^{26}$ To investigate the efficacy of donepezil for prevention of psychosis, the time-to-event (development of psychosis) was compared between patients treated with donepezil hydrochloride and those treated with placebo, because survival time analysis is a suitable approach for this purpose. Focusing on hallucinations, illusions and delusions, psychosis was monitored by a Parkinson's Psychosis Questionnaire (PPQ) every 4 weeks, and the diagnosis of psychosis was based on the criteria predefined by the PPQ scores. PPQ consists of four categories: early symptoms/sleep disturbances (PPQ-A), hallucinations/illusions (PPQ-B), delusions (PPQ-C) and orientation (PPQ-D). The range of each category is $0-9$ points, and the total score ranges from 0 to 36 points. ${ }^{27}$ Decline of cognitive function was investigated by the Mini-Mental State Examination (MMSE) and Wechsler Memory Scale (WMS). Daytime sleepiness was evaluated using the Epworth Sleepiness Scale (ESS), ${ }^{28}$ which is a suitable measure in PD. ${ }^{29}$

\section{Eligibility}

Patients with PD who were $20-80$ years old (inclusive) at the time of signing the consent and that met the PD diagnostic criteria according to steps 1 and 2 of the UK Brain Bank Parkinson's Disease Criteria, with modified Hoehn-Yahr (mH-Y) stage from 2.5 to 4 (during the ON period if patients suffered from motor fluctuations), and with an MMSE score of 24 or more were invited to participate in the trial. Because risk of psychosis is elevated in patients with $\mathrm{mH}-\mathrm{Y} 4$, those patients without dementia were also invited to participate. Other inclusion criteria included lack of psychosis at study entry, and PPQ-B (hallucinations/illusions) and PPQ-C (delusions) scores of zero for at least 8 weeks before study enrolment. If PPQ-B and PPQ-C scores were zero for at least 8 weeks before study entry, patients who had had psychosis were also included. The inclusion criteria did not take into consideration the scores of PPQ-A (early features/ sleep disorders) or PPQ-D (orientation/recent memory).

The study excluded patients who had been treated with donepezil or anticholinergic drugs in the preceding 4 weeks, with antipsychotic drugs in the preceding 12 weeks, or fulfilled the criteria of probable DLB according to the third report of the DLB consortium..$^{30}$ Patients who had been diagnosed with schizophrenia or undergone stereotactic brain surgery were also excluded. Those with allergy to piperidine derivatives, severe hepatic or renal dysfunction, sick sinus syndrome or cardiac conduction block in the atrium, sinoatrial block or atrioventricular block of $2^{\circ}$ or more or had severe bronchial asthma, severe peptic ulcers or severe obstructive pulmonary disorders were also excluded. Patients with a heart rate less than 45/min or QT time $>460$ ms on an ECG were also excluded at screening, as were pregnant women or patients with malignancy. The purpose and methods were explained, and signed consent was obtained.

\section{Procedures}

Screening for eligibility was performed, and written informed consent was obtained from candidate participants at the first study visit (V1), and eligibility was confirmed at the second study visit (V2). Participants were randomly assigned to receive donepezil hydrochloride or placebo, and observation began at V2. Randomisation was performed by a centralised web-based service with a dynamic allocation for sites, $\mathrm{mH}-\mathrm{Y}$ stage (2.5-3 vs 4), sex, and history of psychosis. The investigational drug ( $3 \mathrm{mg} /$ day donepezil) or placebo was started at V3 (2 weeks from the start of observation), and the dose was escalated to $5 \mathrm{mg} /$ day at V4 (4 weeks from the start of the observation), according to the standard dosages approved for Alzheimer's disease by the Pharmaceuticals and Medical Devices Agency in Japan. Donepezil or placebo was prescribed in the morning. The allocation was generated by a computer and concealed by a key-code holder (KK). The tablets of both donepezil and placebo were manufactured by Eisai Pharmaceutical Company, Japan, and were physically indistinguishable, as confirmed by KK, who did not have any other roles in the study.

\section{Evaluation and definition of psychosis}

To detect the development of psychosis including hallucinations, illusions and delusions, patients or their caregivers kept a psychosis diary that had been pre-prepared by the investigators. Every 4 weeks, the PPQ was performed, the psychosis diary was checked and the participants and caregivers were interviewed. The development of psychosis was defined as PPQ-B (hallucinations or illusions) $\geq 2$ points or $\mathrm{PPQ}-\mathrm{C}$ (delusions) $\geq 2$ points, because psychosis with these scores was considered clinically relevant. When the scores of PPQ-B or PPQ-C were $\geq 2$ points, the onset of symptoms was determined by checking the diary and by interviewing patients and caregivers. In case of inconsistency between the diary and interviews, the earlier date was considered the onset date.

\section{Outcome measures}

The primary outcome measure, which was the time to the first development of psychosis from randomisation, that is, the beginning of study observation (V2), was compared between the donepezil and placebo groups. The secondary outcome measures included changes in total PPQ scores and cognitive function, which was evaluated by MMSE, WMS and the Frontal Assessment Battery (FAB).

PPQ was performed every 4 weeks, while mH-Y, Unified Parkinson's Disease Rating Scale Part 3 (UPDRS-III) and ESS were conducted every 8 weeks. MMSE and WMS were conducted at $\mathrm{V} 1$ and $\mathrm{V} 2$, respectively, while $\mathrm{FAB}$ was performed in the period between V2 and V3. MMSE, WMS and FAB were repeated at V9 (24 weeks), V15 (48 weeks), V21 (72 weeks) and V27 (96 weeks).

\section{Apolipoprotein E subgroup analysis}

Because the response to donepezil is modified by apolipoprotein E genotypes in Alzheimer disease, ${ }^{31}$ we analysed outcome measures in subgroups according to the $\varepsilon 4$ allele. 


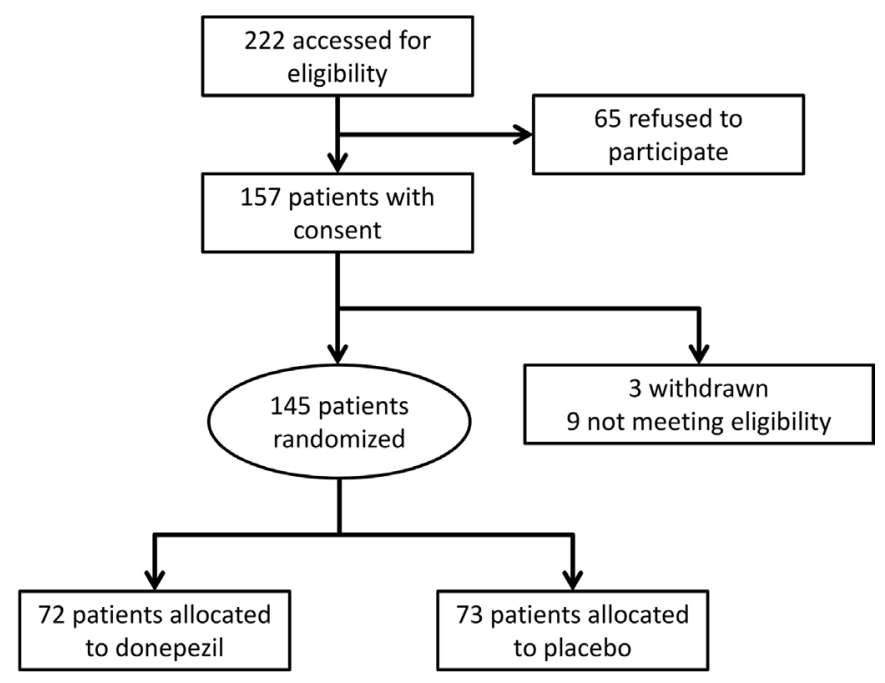

Figure 1 Flow diagram of the patient recruitment process.

\section{Sample size calculation}

In our previous study that followed 258 patients with PD over 2 years, $51(19.7 \%)$ patients had psychosis requiring antipsychotic medication. ${ }^{23}$ We reanalysed the rate of psychosis requiring antipsychotic medication from our previous study according to the eligibility criteria described above, and we assumed that the cumulative occurrence of psychosis was $45 \%$ in the placebo group, which was reduced to $22.5 \%$ in the donepezil group. The sample size was calculated with the conditions: $\alpha=0.05$ (bilateral), power $=0.8$ and using the log-rank test.

$$
\begin{aligned}
\theta= & \frac{\log (0.775)}{\log (0.55)}=0.426 \\
e \equiv & \left(\frac{\theta+1}{\theta-1}\right)^{2}\left(Z_{\frac{\alpha}{2}}+Z_{\beta}\right)=\left(\frac{1.426}{0.574}\right)^{2} \\
& \times(1.960+0.842)^{2}=48.422 \cong 48 \\
n= & \left(\frac{e}{2-0.775-0.55}\right)=71.1 \cong 71
\end{aligned}
$$

We assumed that 48 events were required and calculated a sample size of 142 (71 in each group) patients to provide $80 \%$ power with a $5 \%$ significance level for a log-rank test. ${ }^{26}$

\section{Statistical analysis}

The primary outcome measure, time from randomisation to the development of psychosis, was compared between the two treatment groups by a Cox proportional hazard model that incorporated possible confounding factors. To identify confounding factors, Kaplan-Meier curves were drawn, dividing patients into two groups by factors such as age, sex, PD duration, PD severity, daytime sleepiness and psychosis history, and the effect size of interventions was estimated as an HR after adjustment of the identified confounding factors. If no confounding factors were identified, the difference was compared by a log-rank test. Missing data were regarded as censored but not supplemented in survival analysis.

Secondary outcome measures, changes in PPQ, MMSE, WMS and FAB scores at V9, V15, V21 and V27 from baseline were compared. The data were statistically tested, and the effect size was estimated using a generalised estimating equation (GEE) with an M-based working matrix because they were measured repeatedly every 24 weeks. Generalised linear models assume that individual data in a group are independent from one another; however, in cluster analysis, this assumption often fails. In contrast to generalised linear models, GEE is a statistical method to deal with longitudinal and clustered (non-independent) data. ${ }^{32}$ Missing data were not supplemented in GEE. Data were analysed using IBM SPSS V.21.

\section{Safety analysis}

For safety analysis, adverse effects in the two groups were collected. In addition, we investigated the changes in UPDRS-III and in dose of dopaminergic medications, which were statistically compared using a GEE. The dose of dopaminergic drugs was calculated according to a previous report. ${ }^{33}$

\section{RESULTS}

\section{Baseline features of study subjects}

A total of 222 candidate patients who had been diagnosed with PD and treated in the eight hospitals of the National Hospital Organization were invited to participate in the study. Sixty-five patients refused to participate, and the remaining 157 provided written informed consent, but three patients withdrew and nine were confirmed ineligible at V2 because their PPQ-B or PPQ-C scores were not zero. The remaining 145 patients (51-80 years old) were randomised, and 72 and 73 patients were allocated to donepezil hydrochloride and placebo groups, respectively (figure 1). The baseline features of the study participants are shown in table 1. Age, sex and PD duration were almost identical between the groups.

\begin{tabular}{|c|c|c|}
\hline & $\begin{array}{l}\text { Donepezilgroup } \\
(\mathrm{N}=72)\end{array}$ & $\begin{array}{l}\text { Placebogroup } \\
(\mathrm{N}=73)\end{array}$ \\
\hline Age,year, mean (SD) & $67.2(7.3)$ & $69.0(7.0)$ \\
\hline Female sex, n (\%) & $41(57)$ & $39(53)$ \\
\hline PD duration, year, mean (SD) & $8.2(4.8)$ & $7.8(4.3)$ \\
\hline \multicolumn{3}{|l|}{ Modified H-Y, n (\%) } \\
\hline 2.5 & $36(50)$ & $38(52)$ \\
\hline 3 & $25(35)$ & $29(40)$ \\
\hline 4 & $11(15)$ & $6(8)$ \\
\hline MMSE, mean (SD) & $27.8(1.9)$ & $27.7(2.1)$ \\
\hline History of psychosis, $n(\%)$ & $25(35)$ & $25(34)$ \\
\hline ESS, mean (SD) & $7.0(5.0)$ & $6.7(5.3)$ \\
\hline \multicolumn{3}{|l|}{ UPDRS, mean (SD) } \\
\hline Part I & $0.8(1.0)$ & $0.6(0.9)$ \\
\hline Part II ON & $6.6(5.2)$ & $6.2(4.8)$ \\
\hline Part II OFF & $13.7(7.0)$ & $12.2(6.9)$ \\
\hline Part III & $21.4(11.0)$ & $21.7(8.9)$ \\
\hline Part IV & $3.2(2.5)$ & $2.6(2.3)$ \\
\hline \multicolumn{3}{|l|}{ WMS, mean (SD) } \\
\hline Auditory & $49.8(18.8)$ & $45.0(19.2)$ \\
\hline Visual & $48.8(10.8)$ & $46.1(12.0)$ \\
\hline Attention & $57.1(11.6)$ & $56.3(8.5)$ \\
\hline Delayed recall & $59.0(19.2)$ & $53.3(22.7)$ \\
\hline $\mathrm{FAB}$, mean $(\mathrm{SD})^{*}$ & $14.6(2.4)$ & $13.8(2.1)$ \\
\hline LDED (mg/day) & $640.8(244.1)$ & $624.6(240.5)$ \\
\hline Dopamine agonist user, n (\%) & $48(66.7)$ & $52(71.2)$ \\
\hline Amantadine user, $\mathrm{n}(\%)$ & $18(25.0)$ & $15(20.5)$ \\
\hline Apolipoprotein E4, n (\%)† & $21(34.4)$ & $16(25.0)$ \\
\hline \multicolumn{3}{|c|}{$\begin{array}{l}\text { * }{ }^{*}=70 \text { (donepezil), } 73 \text { (placebo),p=0.045. } \\
\mathrm{tn}=61 \text { (donepezil), } 64 \text { (placebo). } \\
\text { ESS,Epworth Sleepiness Scale;FAB,Frontal Assessment Battery;H-Y,Hoehn- } \\
\text { Yahr;LDED,L-Dopa equivalent dose of dopaminergic replacement } \\
\text { therapy;MMSE,Mini-Mental State Examination;PD,Parkinson's } \\
\text { disease;WMS,Wechsler Memory Scale. }\end{array}$} \\
\hline
\end{tabular}
The proportion of patients with $\mathrm{mH}-\mathrm{Y} 4$ was higher in the donepezil group, although the difference was not significant. MMSE, ESS, UPDRS and WMS scores were also similar. There was a slight 
but significant difference in FAB score, and it was higher in the donepezil than the placebo group. The prevalence of patients on dopamine agonists or amantadine was similar. The prevalence of apolipoprotein $\varepsilon 4$ was higher in the donepezil than in the placebo group, but it was not significantly different. There were no differences between sites in patient characteristics.

\section{Primary outcome}

Forty-six patients developed predefined psychosis during the study. Sample size calculation assumed 48 events, and the statistical assumption was well accepted. The survival curves of psychosis-free patients showed no difference between the donepezil and placebo groups (figure $2 \mathrm{~A}$ ). The effect of potential confounding predictors (age, sex, PD duration, PD severity, sleepiness and psychosis history) was assessed using Kaplan-Meier curves. There were no differences in survival curves according to age (using median age as the cut-off value, $<68$ and $\geq 68$ ), sex or PD duration ( $<8$ years and $\geq 8$ years). In contrast, patients with severe PD, high ESS scores or psychosis history were significantly more prone to develop psychosis (data not shown), and therefore, these factors were included in Cox proportional

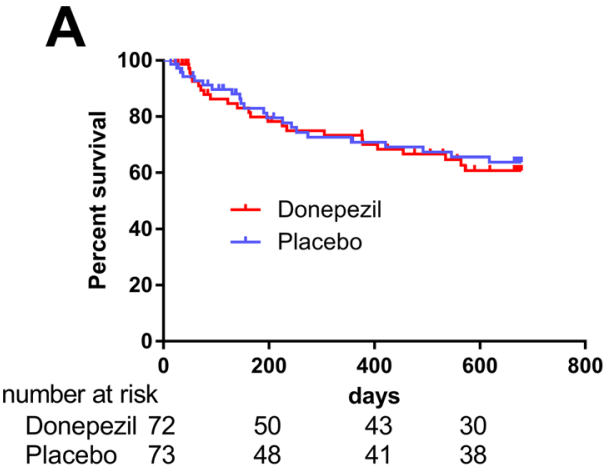

B

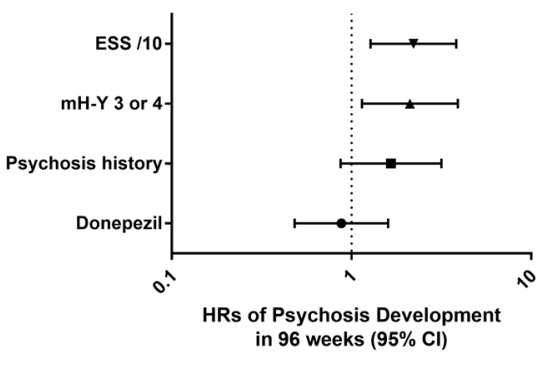

\section{C}

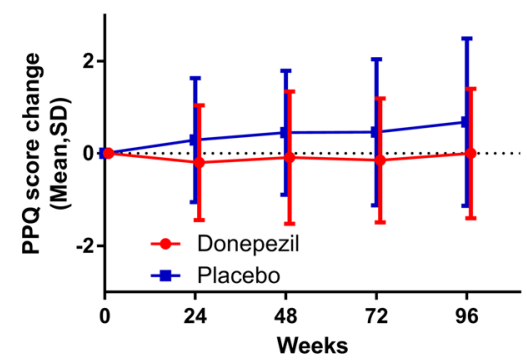

D
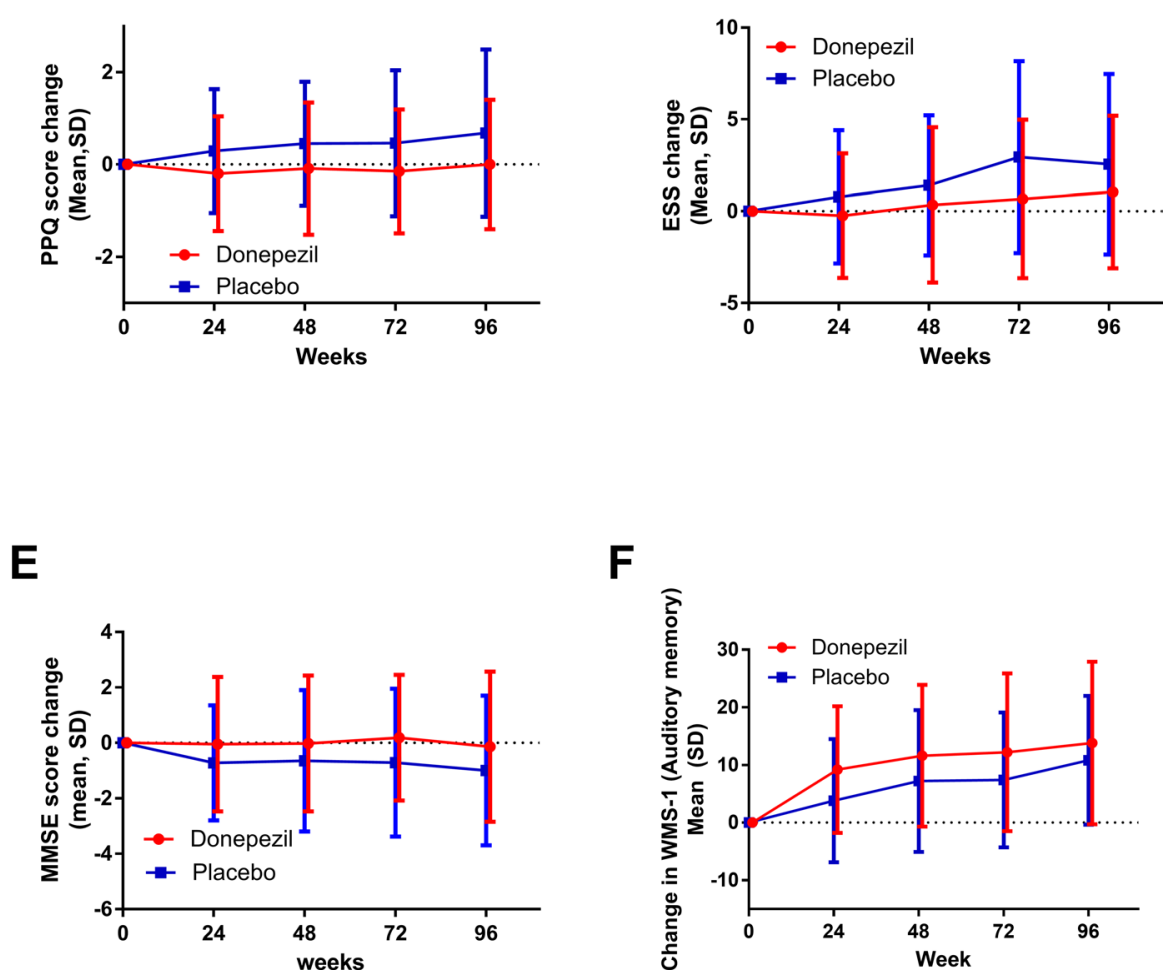

Figure 2 Primary ( $A$ and $B$ ) and secondary outcome $(C-E)$ measures. $(A)$ The survival curves of psychosis-free patients in the donepezil and placebo groups are shown. The number below the horizontal axis shows the number of patients at risk. Censored case is indicated as vertical lines. (B) HR of psychosis was expressed as a mean with 95\% Cls. The horizontal axis has a logarithmic scale. (C) Score changes of PPQ from baseline at V9, V15, V21 and V27, secondary outcomes. Data are expressed as mean and SD. There was a statistically significant difference between donepezil and placebo ( $p=0.004, \mathrm{GEE})$. (D) Changes in ESS scores from baseline at V9, V15, V21 and V27, secondary outcomes, expressed as mean and SD. There was a statistically significant difference $(p=0.042$, GEE). (E) Changes in MMSE from baseline at V9, V15, V21 and V27, secondary outcomes, expressed as mean and SD. There was a statistically significant difference ( $p=0.032$, GEE). (F) Changes in WMS-1 (auditory memory) at V9, V15, V21 and V27, secondary outcomes, expressed as mean and SD. There was a statistically significant difference $(p=0.029$, GEE). ESS, Epworth Sleepiness Scale; GEE, generalised estimating equation; $\mathrm{mH}-\mathrm{Y}$, modified HoehnYahr; MMSE, Mini-Mental State Examination; PPQ, Parkinson's Psychosis Questionnaire; WMS, Wechsler Memory Scale. 
Table 2 Secondary outcome measures

\begin{tabular}{|c|c|c|c|c|c|}
\hline & \multirow{2}{*}{$\begin{array}{l}\text { Donepezil } \\
\text { Means (SEM) }\end{array}$} & \multirow{2}{*}{$\begin{array}{l}\text { Placebo } \\
\text { Means (SEM) }\end{array}$} & \multicolumn{2}{|c|}{ Treatment effect* } & \multirow[b]{2}{*}{$P$ values } \\
\hline & & & Means & $(95 \% \mathrm{Cl})$ & \\
\hline \multicolumn{6}{|l|}{ Psychosis } \\
\hline Cx in total PPQ & $-0.12(0.152)$ & $0.53(0.166)$ & -0.652 & $(-1.095$ to -0.209$)$ & 0.004 \\
\hline \multicolumn{6}{|l|}{ Sleepiness } \\
\hline Cx in ESS & $0.36(0.45)$ & $1.69(0.47)$ & -1.33 & $(-2.61$ to -0.05$)$ & 0.042 \\
\hline \multicolumn{6}{|l|}{ Cognitive function } \\
\hline Cx in scores of MMSE & $0.02(0.28)$ & $-0.78(0.25)$ & 0.8 & (0.07 to 1.54 ) & 0.032 \\
\hline Cx in WMS-1 (auditory memory) & $11.2(1.48)$ & $7.06(1.20)$ & 4.15 & (0.43 to 7.87$)$ & 0.029 \\
\hline Cx in WMS-2 (visual memory) & $3.85(0.66)$ & $3.32(0.80)$ & 0.53 & $(-1.50$ to 2.56$)$ & 0.609 \\
\hline Cx in WMS-4 (attention) & $1.02(0.87)$ & $0.92(0.97)$ & 0.1 & ( -2.46 to 2.66$)$ & 0.94 \\
\hline$C x$ in $F A B$ & $0.07(0.22)$ & $0.39(0.23)$ & -0.32 & $(-0.94$ to 0.32$)$ & 0.315 \\
\hline
\end{tabular}

Higher scores in PPQand ESS are worse.

Higher scores in MMSE, WMS and FAB are better.

*Effect size was estimated using GEE (working matrix M-dependent).

Cx: changes; ESS, Epworth Sleep Scale; FAB, Frontal Assessment Battery; GEE, generalised estimating equation; MMSE, Mini-Mental State Examination; PPQ, Parkinson's Psychosis Questionnaire; WMS, Wechsler Memory Scale.

hazard models. The HR of psychosis was 0.87 (95\% CI 0.48 to 1.60 ) with adjustment for psychosis history, PD severity and sleepiness (figure 2B). The primary outcome measure was similar between females and males (online supplementary table 1).

\section{Secondary outcome}

Total PPQ scores elevated gradually throughout the study period in the placebo group but were stable in the donepezil group, and the difference was statistically significant (figure 2C). As well as PPQ scores, ESS scores increased gradually in the placebo group but were stable in the donepezil group, with statistical significance (figure 2D).

The score changes in MMSE with donepezil were better than placebo, and there was statistical significance (figure $2 \mathrm{E}$ ). Changes in WMS-1 were significantly better with donepezil (figure 2F), but visual memory (WMS-2), attention (WMS-3) and frontal lobe function (FAB) were not affected by the intervention. The effect sizes are shown in table 2. Changes in PPQ scores from baseline were significantly better in donepezil than placebo in both sexes (online supplementary tables 2 and 3), suggesting that prophylactic effect of donepezil is almost similar in females and males.

\section{Subgroup analysis by apolipoprotein E genotype}

In the subgroup of $\varepsilon 4$ non-carriers, Kaplan-Meier curves split at 48 weeks and joined at 96 weeks (figure 3A). Cox proportional hazard models demonstrated a significant prophylactic effect with a HR of $0.31(0.11-0.86, p=0.02)$ against psychosis development in 48 weeks (figure 3C), although the difference was not significant against psychosis development in 96 weeks (HR was $0.63(0.28-1.41, p=0.26)$ ). In this subgroup, there was a significant difference between donepezil and placebo for changes in total PPQ scores (figure 3E) and changes in ESS scores (figure $3 \mathrm{G}$ ).

In $\varepsilon 4$ carriers, in contrast to the $\varepsilon 4$ (-) subgroup, KaplanMeier curves were similar between donepezil and placebo (figure $3 \mathrm{~B})$, and the adjusted HR was 0.85 (95\% CI 0.19 to 3.79 , $\mathrm{p}=0.84$; figure $3 \mathrm{D})$ and $0.86(95 \%$ CI 0.23 to $3.32, \mathrm{p}=0.83)$ for psychosis in 48 and 96 weeks, respectively. There was no difference in PPQ and ESS changes in the subgroup (figure $3 \mathrm{~F}$ and $\mathrm{H}$ ). The effect sizes of donepezil in each subgroup are demonstrated in table 3 .

\section{Safety analysis}

The most frequent adverse event was visual hallucinations; 39 events occurred in the donepezil group and 36 in the placebo group. In addition, other types of hallucinations and delusions were also often observed. The prevalence of hallucinations and delusions was also similar in the donepezil and placebo groups. However, auditory hallucinations were less prevalent in the donepezil than in the placebo group (table 4). Worsening Parkinsonism was observed more frequently in the donepezil (12 events) than the placebo group (six events), and motor complications were also more often observed in the donepezil group. Falls and contusion injuries were observed often in both groups. Nausea, vomiting, loss of appetite and diarrhoea were more common with donepezil than with placebo. Body weight loss was observed only in patients in the donepezil group.

The effects on motor symptoms and signs were investigated by UPDRS-III. Since dopamine replacement therapy was not restricted, the UPDRS-III was evaluated considering the changes in dosage of dopamine replacement. In the donepezil group, the mean UPDRS-III scores increased at 16-24 weeks after randomisation but returned to the baseline at 32 weeks and remained stable thereafter. In the placebo group, the UPDRS-III scores were stable throughout the study. The mean difference in UPDRS-III was 0.45 (95\% CI -1.25 to $2.15, \mathrm{p}=0.61$; figure $4 \mathrm{~A})$. The dose of dopaminergic replacement therapy increased steadily throughout the study in both groups, but the increase was higher in the donepezil than in the placebo group, with a mean difference of 17.6 (95\% CI, -10.1 to $45.2 \mathrm{mg} / \mathrm{day}$, $\mathrm{p}=0.213$; figure $4 \mathrm{~B}$ ).

Because donepezil can enhance rapid eye movement (REM) sleep and cause nightmares, ${ }^{34}$ a lack of prophylactic effect against psychosis development may be due to nightmares caused by donepezil. In this context, we investigated PPQ-A scores (sleep disturbances including nightmares) and found no difference in PPQ-A scores between the groups; changes in PPQ-A scores were $-0.20(95 \%$ CI -0.37 to -0.03$)$ and $-0.09(95 \%$ $\mathrm{CI}-0.28$ to 0.10 ) in donepezil and placebo groups, respectively.

In a non-predefined subgroup analysis of MMSE $<28$ and MMSE $\geq 28$, donepezil did not provide prophylactic effects against psychosis in either subgroup by survival time analysis. However, changes in PPQ from baseline were significantly better in the donepezil than in the placebo subgroup of MMSE $\geq 28$, 

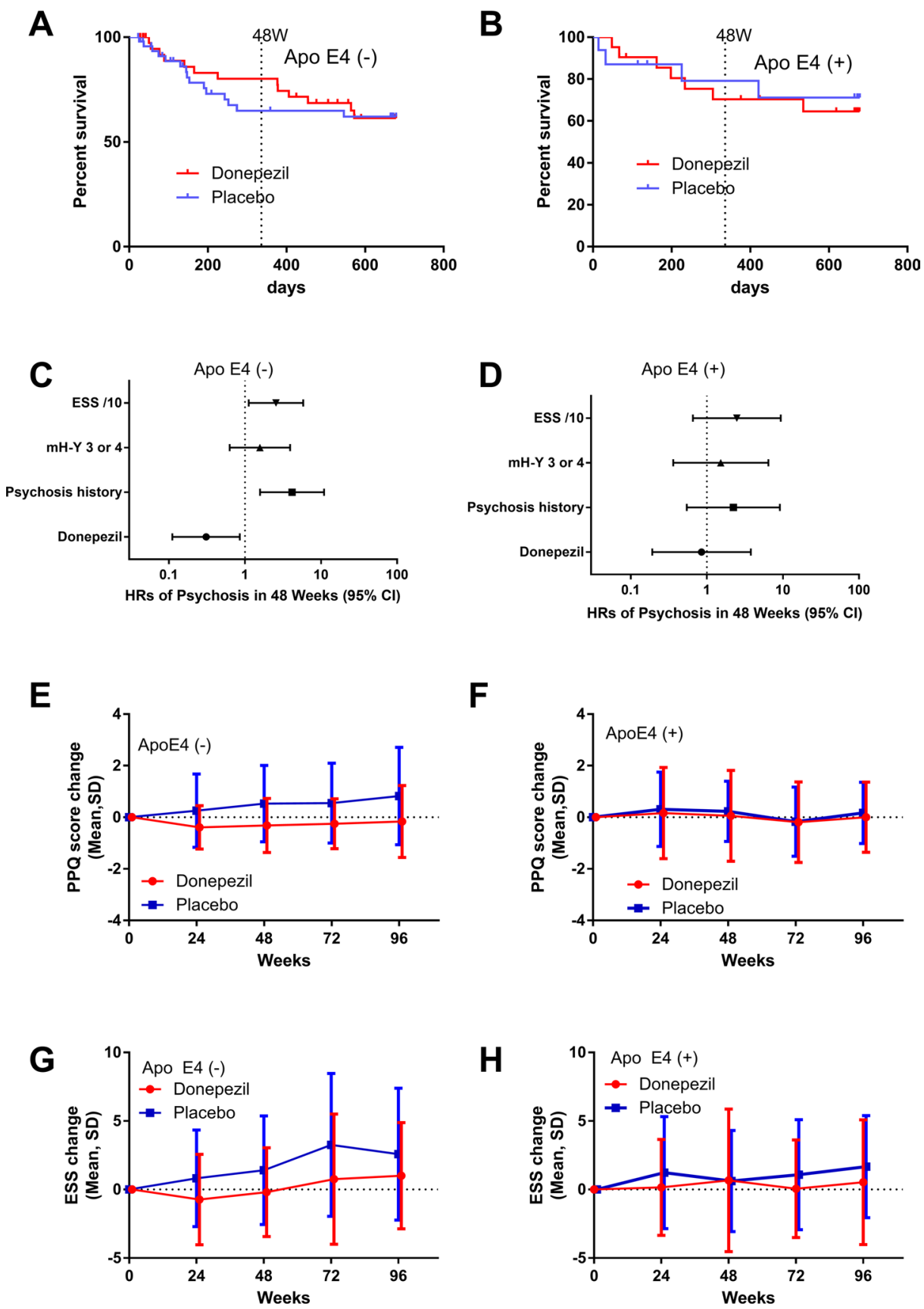

Figure 3 Subgroup analysis by apolipoprotein $\varepsilon 4$ (Apo E4) allele. ( $A$ and B) Kaplan-Meier curves of psychosis-free patients (A) without $\varepsilon 4$ and (B) with the $\varepsilon 4$ allele. The curves separate at 48 weeks in the $\varepsilon 4(-)$ subgroup but not in the $\varepsilon 4(+)$ subgroup. (C and D) Cox proportional hazard models for psychosis development in 48 weeks in patients (C) without $\varepsilon 4$ and (D) with the $\varepsilon 4$ allele. There was a statistically significant difference between donepezil and placebo groups in the $\varepsilon 4$ (-) subgroup ( $H R=0.31(95 \% \mathrm{Cl} 0.11$ to 0.86$), p=0.02)$, but not in the $\varepsilon 4$ (+) subgroup ( $H R=0.85(95 \% \mathrm{Cl} 0.192$ to 3.78$), p=0.84)$. ( $E$ and F) Changes in total PPQ scores in patients (E) without $\varepsilon 4$ and (F) with the $\varepsilon 4$ allele. There was a statistically significant difference between donepezil and placebo groups in the $\varepsilon 4(-)$ subgroup $(p=0.001)$ but not in $\varepsilon 4(+)$ subgroup. $(G$ and $H)$ Change in ESS scores in patients $(G)$ without $\varepsilon 4$ and $(H)$ with the $\varepsilon 4$ allele. There was a statistically significant difference between donepezil and placebo groups in the $\varepsilon 4(-)$ subgroup ( $p=0.027)$ but not in $\varepsilon 4$ (+) subgroup. ESS, Epworth Sleepiness Scale; mH-Y, modified Hoehn-Yahr; PPQ, Parkinson's Psychosis Questionnaire.

but not in that of MMSE $<28$ (online supplementary tables 4 and 5). Therefore, the effect of donepezil against psychosis may be dependent on cholinesterase activity, because it is assumed to be relatively preserved in cognitive-intact patients.

\section{DISCUSSION}

The subjects of this study had mild to moderate PD with a mean disease duration of 8 years, without dementia, although about one-third of them had experienced psychosis. These features were in alignment with the target of the study to investigate the hypothesis that donepezil provides prophylaxis against psychosis in non-demented PD patients. Overall, comparability between the two study groups was ensured. The number of events that occurred in the study was as expected and sufficient for proper evaluation of the efficacy of donepezil in preventing psychosis, and thus, the obtained data fitted into the statistical assumption.

Analysis using Kaplan-Meier curves indicated that donepezil does not prevent the development of psychosis. The results also 
Table 3 Subgroup analysis

\begin{tabular}{|c|c|c|c|c|c|c|c|}
\hline \multirow[b]{2}{*}{ Subgroup } & \multirow[b]{2}{*}{ Outcome measure } & \multirow[b]{2}{*}{ Analysis } & \multirow{2}{*}{$\begin{array}{l}\text { Donepezil } \\
\text { Means (errors) }\end{array}$} & \multirow{2}{*}{$\begin{array}{l}\text { Placebo } \\
\text { Means (errors) }\end{array}$} & \multicolumn{2}{|c|}{ Treatment effect* } & \multirow[b]{2}{*}{$P$ values } \\
\hline & & & & & Means & $(95 \% \mathrm{Cl})$ & \\
\hline \multirow[t]{6}{*}{ e4 (-) } & Psychosis development & & & & & & \\
\hline & HR for psychosis in 48 weeks & Cox & & & 0.31 & (0.11 to 0.86 ) & 0.02 \\
\hline & HR for psychosis in 96 weeks & Cox & & & 0.63 & (0.28 to 1.41$)$ & 0.26 \\
\hline & $\mathrm{Cx}$ in total $\mathrm{PPQ}$ & GEE & $-0.28(0.167)$ & $0.60(0.209)$ & -0.88 & $(-1.41$ to -0.36$)$ & 0.001 \\
\hline & Sleepiness & & & & & & \\
\hline & Cx in ESS & GEE & $0.45(1.93)$ & $1.85(1.93)$ & -1.4 & $(-2.65$ to -0.16$)$ & 0.027 \\
\hline \multirow[t]{6}{*}{ e4 (+) } & Psychosis development & & & & & & \\
\hline & HR for psychosis in 48 weeks & Cox & & & 0.85 & (0.19 to 3.79 ) & 0.84 \\
\hline & HR for psychosis in 96 weeks & Cox & & & 0.86 & (0.23 to 3.32$)$ & 0.83 \\
\hline & Cx in total PPQ & GEE & $0.02(0.30)$ & $0.20(0.331)$ & -0.18 & $(-1.05$ to 0.70$)$ & 0.69 \\
\hline & Sleepiness & & & & & & \\
\hline & Cx in ESS & GEE & $1.26(3.13)$ & $0.51(3.19)$ & 0.75 & $(-0.85$ to 2.35$)$ & 0.36 \\
\hline
\end{tabular}

*Effect size was estimated using GEE (working matrix M-dependent), adjusted for ESS/10, $\mathrm{mH}-\mathrm{Y}$ (2.5 vs 3-4) and psychosis history.

CX, changes; ESS, Epworth Sleep Scale; GEE, generalised estimating equation; mH-Y, modified Hoehn-Yahr; PPQ, Parkinson's Psychosis Questionnaire.

implied that psychosis was associated with PD severity, sleepiness and history of psychosis, consistent with the results of previous studies. $^{23} 35$ As shown in table 1, patients with $\mathrm{mH}-\mathrm{Y}$ 4 were more prevalent and ESS was higher in the donepezil group than with placebo. After adjustment for these factors, the Cox proportional hazard model demonstrated an HR of 0.87 , slightly smaller than 1 , but the effect was not statistically significant. This finding demonstrates that donepezil does not

\begin{tabular}{lcc}
\hline $\begin{array}{l}\text { Table } 4 \\
\text { group) }\end{array}$ & \multicolumn{1}{l}{ Treatment-related adverse events } & (more than 5\% in either \\
\hline & $(\mathbf{n}=72)$ & $\begin{array}{l}\text { Placebo } \\
(\mathbf{n}=73)\end{array}$ \\
\hline Visual hallucinations & $39(54.2)$ & $36(49.3)$ \\
\hline Auditory hallucinations* & $8(11.1)$ & $26(35.6)$ \\
\hline Hallucinations, unspecified & $8(11.1)$ & $7(9.6)$ \\
\hline Delusions & $21(29.2)$ & $19(26.0)$ \\
\hline Cenesthopathy & $2(2.8)$ & $4(5.5)$ \\
\hline Nasopharyngitis & $15(20.8)$ & $20(38.4)$ \\
\hline Parkinsonism (worsening) & $12(16.7)$ & $6(8.2)$ \\
\hline Dyskinesias & $6(8.3)$ & $2(2.7)$ \\
\hline Dystonia & $4(5.6)$ & $1(1.4)$ \\
\hline Contusion & $9(12.5)$ & $8(11.0)$ \\
\hline Fall & $3(4.2)$ & $4(5.5)$ \\
\hline Vomiting & $5(6.9)$ & $4(5.5)$ \\
\hline Nausea & $5(6.9)$ & $2(2.7)$ \\
\hline Loss of appetite & $6(8.3)$ & $1(1.4)$ \\
\hline Diarrhoea & $4(5.6)$ & $2(2.7)$ \\
\hline Loose stool & $4(5.6)$ & $0(0)$ \\
\hline Body weight loss & $4(5.6)$ & $0(0)$ \\
\hline Urinary cystitis & $8(11.1)$ & $13(17.8)$ \\
\hline Dental caries & $5(6.9)$ & $4(5.5)$ \\
\hline Back pain & $4(5.6)$ & $7(9.6)$ \\
\hline Limb pain & $4(5.6)$ & $2(2.7)$ \\
\hline Headache & $6(8.3)$ & $1(1.4)$ \\
\hline Upper respiratory tract infection & $5(6.9)$ & $4(5.5)$ \\
\hline Aspiration pneumonia & $0(0.0)$ & $5(6.8)$ \\
Insomnia & $5(6.9)$ & $5(6.8)$ \\
\hline
\end{tabular}

The number of adverse events: number of events/patients(\%).

*There were statistically significant differences in the prevalence of auditory hallucinations $(p=0.00007)$ and nasopharyngitis $(p=0.0287)$. have significant prophylactic effect against psychosis in non-demented PD patients. In contrast to the results described above, the changes in both total PPQ score and ESS were significantly better in the donepezil group than with placebo, suggesting that prevention of psychosis is a harder endpoint than score changes in total PPQ or ESS.

Because the extent of cognitive improvement with donepezil correlates with brain cholinesterase activity, ${ }^{36}$ the efficacy of donepezil was expected to be best seen in non-demented PD patients, since they are known to have preserved cholinesterase activity, rather than in patients with DLB. As expected, subgroup analysis by lower and higher baseline MMSE demonstrated that donepezil provided beneficial effects in higher MMSE group but not in lower group.

There was no significant difference in FAB scores between donepezil and placebo; however, the result is limited because of a significant difference at baseline. The present study demonstrated that donepezil was efficacious in psychological tests for cognitive function evaluated by WMS. In this regard, several previous randomised control studies showed that donepezil improves cognitive function in PD with dementia. ${ }^{37} 38$ Dubois and colleagues ${ }^{39}$ conducted a large-scale randomised control trial to investigate the effects of donepezil on cognition in PD with dementia and confirmed that donepezil improves cognitive function; however, their results showed no significant changes in psychiatric symptoms. These results suggest that while cognitive psychological test scores can be improved by donepezil, the effects on psychiatric symptoms are difficult to be detected because psychiatric symptoms can occur transiently and fluctuate. The effect size of MMSE changes in the present study was 0.80 , which is clinically irrelevant, and slightly smaller than that reported previously. ${ }^{40}$ This is thought to be due to differences in clinical features of the study participants, especially differences in baseline MMSE scores. In the present study, the baseline scores were higher than in previous studies, and therefore the effect size was undervalued.

In Alzheimer's disease, the response of cognitive dysfunction to donepezil is greater in apolipoprotein $\varepsilon 4$ non-carriers. ${ }^{41}$ In subgroup analysis for apolipoprotein $\varepsilon 4$, donepezil provided significant prophylactic effect against psychosis development in 48 weeks in $\varepsilon 4$ non-carriers, but not in $\varepsilon 4$ carriers. In addition, donepezil modulated the changes in total PPQ scores and ESS in $\varepsilon 4$ non-carriers but not in carriers, suggesting that apolipoprotein 
A

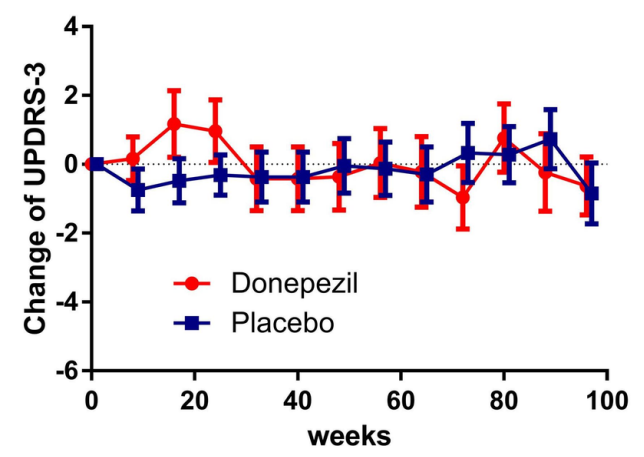

B

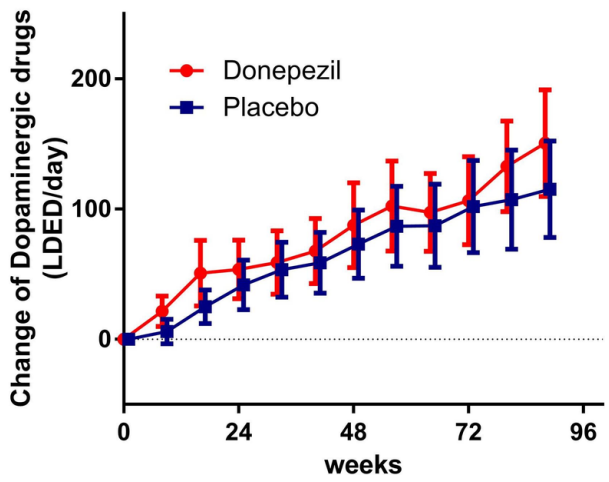

Figure 4 Changes in UPDRS-III scores and L-Dopa equivalent dose of dopaminergic replacement therapy (LDED). (A) In the donepezil group, UPDRSIII scores were transiently elevated by starting and dose escalation (3-5 mg/day) of donepezil, but then returned to baseline and remained stable. In the placebo group, the scores were stable. There were no statistically significant differences in UDPRS-III changes between donepezil and placebo groups. (B) Dopaminergic drug doses were elevated gradually in both donepezil and placebo groups and were slightly larger with donepezil than with placebo. The difference was not statistically significant. Data represent the mean with $95 \% \mathrm{Cls}$.

E4 is a suppressor for psychosis prophylaxis. Apolipoprotein $\varepsilon 4$ carriers are prone to develop dementia and the risk of hallucinations is significantly higher, ${ }^{42}{ }^{43}$ although the association is controversial. ${ }^{44}$ These data suggest that apo E4 carriers may be more likely to have a comorbid amyloid burden causing cortical dysfunction that prevents donepezil from being as effective.

Previous reports showed that donepezil does not worsen extrapyramidal signs and symptoms. ${ }^{40}$ In the present study, while the UPDRS-III scores were not significantly different between donepezil and placebo, the scores of the donepezil group worsened transiently and decreased, most likely owing to increases in the dosage of dopamine replacement therapy. Thus, to maintain UPDRS-III scores, a larger dosage of dopamine replacement therapy was required in the donepezil group.

Although the effects of donepezil, at least at $5 \mathrm{mg} /$ day, were weak and inadequate to provide prophylaxis against the development of psychosis in patients with PD, early use of donepezil improved the scores for PPQ, MMSE and auditory WMS significantly for 2 years in cognitive preserved patients, without worsening of motor symptoms. These results suggest that early use of donepezil may be efficacious against cognitive decline but not for prophylaxis of psychosis.

\section{Author affiliations \\ ${ }^{1}$ Clinical Research Center and Department of Neurology, Utano National Hospital, Kyoto, Japan \\ ${ }^{2}$ Department of Neurology, Shizuoka Medical Institute of Epilepsy and Neurological Disorders, Shizuoka City, Japan \\ ${ }^{3}$ Department of Neurology, Nagasaki Kawatana Medical Center, Nagasaki, Japan \\ ${ }^{4}$ Department of Neurology, Sagamihara National Hospital, Sagamihara, Japan \\ ${ }^{5}$ Clinical Research Center and Department of Neurology, Toneyama National Hospital, Toyonaka, Japan \\ ${ }^{6}$ Department of Neurology, Minami-Kyoto National Hospital, Joyo, Japan \\ ${ }^{7}$ Department of Neurology, Kyoto Medical Center, Kyoto, Japan \\ ${ }^{8}$ Department of Neurology, Hokkaido Medical Center, Sapporo, Japan \\ ${ }^{9}$ Clinical Research Center, National Hospital Organization, Meguro, Japan \\ ${ }^{10}$ Division of General Internal Medicine, Kyoto Medical Center, Kyoto, Japan \\ ${ }^{11}$ Department of Public Health, Tokyo Women's Medical University, Shinjuku-ku, Japan \\ ${ }^{12}$ Health Center, Kyoto University, Kyotoi, Japan}

Correction notice Since this article was first published online the author $\mathrm{Dr}$ Kukuchi has been updated. The surname now reads Kikuchi.

Acknowledgements We thank neurologists of the National Hospital Organization, Japan: M Niino, MD, PhD (Hokkaido Medical Center); T Obi, MD, PhD (Shizuoka Medical Institute of Epilepsy and Neurological Disorders); R Motoyama, MD and H Nakano, MD (Utano National Hospital); R Ohtani, MD, PhD (Kyoto Medical
Center); N Oka, MD, PhD (Minami-Kyoto National Hospital); and T Nakane, MD, PhD and T Fukutome, MD, PhD (Nagasaki Kawatana Medical Center). We also thank Yuko Mori, BA (Utano National Hospital) for her technical assistance in apolipoprotein genotyping, and Kyoko Waki, BSc (Utano National Hospital) and Kazumi Nakamura BSc (National Hospital Organization) for their help in the study. These collaborators did not receive any compensation for their contributions. We would like to thank Ann Turnley, PhD, from Edanz Group (www.edanzediting.com/ac) for editing a draft of this manuscript.

Contributors HS had full access to all the data in the study and takes responsibility for the integrity of the data and the accuracy of the data analysis. Study concept and design: HS and TO. Acquisition of data: HS, TO, MK, AU, ST, KP, KM, HM, KH, HF, HS, MN and SK. Analysis and interpretation of data: HS, TO, MK, AU, ST, KP, KM, HM, KH, $\mathrm{HF}, \mathrm{HS}, \mathrm{MN}, \mathrm{SK}$, TF and SI. Drafting of the manuscript: HS and TO. Critical revision of the manuscript for important intellectual content: $\mathrm{HS}, \mathrm{TO}, \mathrm{HF}, \mathrm{MN}$ and TK. Statistical analysis: HS, TO, MG and TK. Administrative, technical or material support: TF, KK and SI. Study supervision: HS, TO and SI.

Funding The study was registered with the University hospital Medical Information Network Clinical Trial Registry (UMIN-CTR) (UMIN000005403) and the Pharmaceutical and Medical Devices Agency, Japan (\#22-4018) and approved by the National Hospital Organization Central Review Board (NHO-CRB, Tokyo, Japan). The study was supported by a Grant for the Designated Clinical Research from the National Hospital Organization, Tokyo, Japan. Eisai Pharmaceutical Company supplied donepezil hydrochloride tablets (3 $\mathrm{mg}$ and $5 \mathrm{mg}$ ) and placebo tablets (3 $\mathrm{mg}$ and $5 \mathrm{mg}$ ) in the study. No salaries or stipends were paid. The trial drugs were donated by Eisai Pharmaceutical Company.

Competing interests None declared.

Patient consent Obtained.

Ethics approval The National Hospital Organization Central Review Board, Tokyo, Japan (NHO-CRB).

Provenance and peer review Not commissioned; externally peer reviewed.

Open access This is an open access article distributed in accordance with the Creative Commons Attribution Non Commercial (CC BY-NC 4.0) license, which permits others to distribute, remix, adapt, build upon this work non-commercially, and license their derivative works on different terms, provided the original work is properly cited, appropriate credit is given, any changes made indicated, and the use is non-commercial. See: http://creativecommons.org/licenses/by-nc/4.0/

\section{REFERENCES}

1 Friedman JH. Parkinson disease psychosis: Update. Behav Neurol 2013;27:469-77.

2 Fénelon G, Mahieux F, Huon R, et al. Hallucinations in Parkinson's disease: prevalence, phenomenology and risk factors. Brain 2000;123:733-45.

3 Aarsland D, Larsen JP, Cummins JL, et al. Prevalence and clinical correlates of psychotic symptoms in Parkinson disease: a community-based study. Arch Neurol 1999;56:595-601.

4 Mack J, Rabins P, Anderson K, et al. Prevalence of psychotic symptoms in a community-based Parkinson disease sample. Am J Geriatr Psychiatry 2012;20:123-32.

5 Forsaa EB, Larsen JP, Wentzel-Larsen T, et al. A 12-year population-based study of psychosis in Parkinson disease. Arch Neurol 2010;67:996-1001. 
6 Narayanan NS, Rodnitzky RL, Uc EY. Prefrontal dopamine signaling and cognitive symptoms of Parkinson's disease. Rev Neurosci 2013;24:267-78.

7 Goldman JG, Holden S. Treatment of psychosis and dementia in Parkinson's disease. Curr Treat Options Neurol 2014;16:281.

8 Clozapine in drug-induced psychosis in Parkinson's disease. The French Clozapine Parkinson Study Group. Lancet 1999;353:2041-2.

9 Parkinson Study Group. Low-dose clozapine for the treatment of drug-induced psychosis in Parkinson's disease. N Eng/ J Med 1999:340:757-63.

10 Breier A, Sutton VK, Feldman PD, et al. Olanzapine in the treatment of dopamimetic-induced psychosis in patients with Parkinson's disease. Biol Psychiatry 2002:52:438-45.

11 Ondo WG, Tintner R, Voung KD, et al. Double-blind, placebo-controlled, unforced titration parallel trial of quetiapine for dopaminergic-induced hallucinations in Parkinson's disease. Mov Disord 2005;20:958-63.

12 Rabey JM, Prokhorov T, Miniovitz A, et al. Effect of quetiapine in psychotic Parkinson's disease patients: a double-blind labeled study of 3 months' duration. Mov Disord 2007;22:313-8.

13 Shotbolt P, Samuel M, Fox C, et al. A randomized controlled trial of quetiapine for psychosis in Parkinson's disease. Neuropsychiatr Dis Treat 2009:5:327-32.

14 Ellis T, Cudkowicz ME, Sexton PM, et al. Clozapine and risperidone treatment of psychosis in Parkinson's disease. J Neuropsychiatry Clin Neurosci 2000;12:364-9.

15 Zoldan J, Friedberg G, Livneh M, et al. Psychosis in advanced Parkinson's disease: treatment with ondansetron, a 5-HT3 receptor antagonist. Neurology 1995;45:1305-8.

16 Cummings J, Isaacson S, Mills R, et al. Pimavanserin for patients with Parkinson's disease psychosis: a randomised, placebo-controlled phase 3 trial. Lancet 2014;383:533-40.

17 Meltzer HY, Mills R, Revell S, et al. Pimavanserin, a serotonin(2A) receptor inverse agonist, for the treatment of parkinson's disease psychosis. Neuropsychopharmacology 2010;35:881-92.

18 Manganelli F, Vitale C, Santangelo G, et al. Functional involvement of central cholinergic circuits and visual hallucinations in Parkinson's disease. Brain 2009;132(Pt 9):2350-5.

19 Hilker R, Thomas AV, Klein JC, et al. Dementia in Parkinson disease: functional imaging of cholinergic and dopaminergic pathways. Neurology 2005;65:1716-22.

20 Bosboom JL, Stoffers D, Wolters ECh. The role of acetylcholine and dopamine in dementia and psychosis in Parkinson's disease. J Neural Transm Suppl 2003;65:185-95.

21 Williams-Gray CH, Foltynie T, Lewis SJ, et al. Cognitive deficits and psychosis in Parkinson's disease: a review of pathophysiology and therapeutic options. CNS Drugs 2006:20:477-505.

22 Saint-Cyr JA, Taylor AE, Lang AE. Neuropsychological and psychiatric side effects in the treatment of Parkinson's disease. Neurology 1993;43(12 Suppl 6):S47-52.

23 Sawada H, Oeda T, Yamamoto K, et al. Trigger medications and patient-related risk factors for Parkinson disease psychosis requiring anti-psychotic drugs: a retrospective cohort study. BMC Neurol 2013;13:145.

24 Satoh M, Ishikawa H, Meguro K, et al. Improved visual hallucination by donepezil and occipital glucose metabolism in dementia with Lewy bodies: the Osaki-Tajiri project. Eur Neurol 2010;64:337-44.
25 Klein JC, Eggers C, Kalbe E, et al. Neurotransmitter changes in dementia with Lewy bodies and Parkinson disease dementia in vivo. Neurology 2010;74:885-92.

26 Sawada H, Oeda T. Protocol for a randomised controlled trial: efficacy of donepezil against psychosis in Parkinson's disease (EDAP). BMJ Open 2013;3:e003533.

27 Brandstaedter D, Spieker S, UIm G, et al. Development and evaluation of the Parkinson Psychosis Questionnaire A screening-instrument for the early diagnosis of drug-induced psychosis in Parkinson's disease. J Neurol 2005;252:1060-6.

28 Johns MW. A new method for measuring daytime sleepiness: the Epworth sleepiness scale. Sleep 1991;14:540-5.

29 Kumar S, Bhatia M, Behari M. Excessive daytime sleepiness in Parkinson's disease as assessed by Epworth Sleepiness Scale (ESS). Sleep Med 2003;4:339-42.

30 McKeith IG, Dickson DW, Lowe J, et al. Diagnosis and management of dementia with Lewy bodies: third report of the DLB Consortium. Neurology 2005;65:1863-72

31 Poirier J, Delisle MC, Quirion R, et al. Apolipoprotein E4 allele as a predictor of cholinergic deficits and treatment outcome in Alzheimer disease. Proc Natl Acad Sci U SA 1995:92:12260-4.

32 Hardin JW, Hilbe JM. Generalized estimating equations. Boca Raton, Florida: CRC press LLC, 2003.

33 Tomlinson CL, Stowe R, Patel S, et al. Systematic review of levodopa dose equivalency reporting in Parkinson's disease. Mov Disord 2010;25:2649-53.

34 Singer $\mathrm{M}$, Romero $\mathrm{B}$, Koenig $\mathrm{E}$, et al. [Nightmares in patients with Alzheimer's disease caused by donepezil. Therapeutic effect depends on the time of intake]. Nervenarzt 2005;76:1127-9.

35 Fénelon G, Alves G. Epidemiology of psychosis in Parkinson's disease. J Neurol Sci 2010;289(1-2):12-17.

36 Hiraoka K, Okamura N, Funaki Y, et al. Cholinergic deficit and response to donepezil therapy in Parkinson's disease with dementia. Eur Neurol 2012;68:137-43.

37 Leroi I, Brandt J, Reich SG, et al. Randomized placebo-controlled trial of donepezil in cognitive impairment in Parkinson's disease. Int J Geriatr Psychiatry 2004;19:1-8.

38 Ravina B, Putt M, Siderowf A, et al. Donepezil for dementia in Parkinson's disease: a randomised, double blind, placebo controlled, crossover study. J Neurol Neurosurg Psychiatry 2005;76:934-9.

39 Dubois B, Tolosa E, Katzenschlager R, et al. Donepezil in Parkinson's disease dementia: a randomized, double-blind efficacy and safety study. Mov Disord 2012;27:1230-8.

40 Aarsland D, Laake K, Larsen JP, et al. Donepezil for cognitive impairment in Parkinson's disease: a randomised controlled study. J Neurol Neurosurg Psychiatry 2002:72:708-12.

41 Choi SH, Kim SY, Na HR, et al. Effect of ApoE genotype on response to donepezil in patients with Alzheimer's disease. Dement Geriatr Cogn Disord 2008;25:445-50.

42 Monsell SE, Besser LM, Heller KB, et al. Clinical and pathologic presentation in Parkinson's disease by apolipoprotein e4 allele status. Parkinsonism Relat Disord 2014;20:503-7.

43 de la Fuente-Fernández R, Núñez MA, López E. The apolipoprotein E epsilon 4 allele increases the risk of drug-induced hallucinations in Parkinson's disease. Clin Neuropharmacol 1999;22:226-30.

44 Factor SA, Steenland NK, Higgins DS, et al. Disease-related and genetic correlates of psychotic symptoms in Parkinson's disease. Mov Disord 2011;26:2190-5. 